\title{
Remarks on a theorem by N. Yu. Antonov
}

\author{
by \\ Per SJölin (Stockholm) and Fernando Soria (Madrid)
}

\begin{abstract}
We extend some results of N. Yu. Antonov on convergence of Fourier series to more general settings. One special feature of our work is that we do not assume smoothness for the kernels in our hypotheses. This has interesting applications to convergence with respect to general orthonormal systems, like the Walsh-Fourier system, for which we prove a.e. convergence in the class $L \log L \log \log \log L$. Other applications are given in the theory of differentiation of integrals.
\end{abstract}

1. Introduction. Let $\mathbb{T}=\mathbb{R} / \mathbb{Z}$ denote the one-dimensional torus, which we identify with the interval $[0,1)$. We set

$$
D_{m}(x)=\frac{\sin (2 m+1) \pi x}{\sin \pi x}, \quad m=0,1, \ldots,
$$

the $m$ th Dirichlet kernel, so that for a function $f \in L^{1}(\mathbb{T})$,

$$
S_{m} f(x)=D_{m} * f(x)
$$

denotes its Fourier $m$ th partial sum. Given $N \in \mathbb{N}$, we define

$$
S_{N}^{*} f(x)=\sup _{0 \leq m \leq N}\left|S_{m} f(x)\right|, \quad S^{*} f(x)=\sup _{m \geq 0}\left|S_{m} f(x)\right| .
$$

$S^{*}$ is called the Carleson maximal operator.

Extending the celebrated theorem of Carleson [4] on the a.e. convergence of Fourier series for functions in $L^{2}(\mathbb{T})$, R. Hunt [7] proved the remarkable inequality for $1<p<\infty$,

$$
\lambda\left|\left\{x \in \mathbb{T}: S^{*} \chi A(x)>\lambda\right\}\right|^{1 / p} \leq C \frac{p^{2}}{p-1}|A|^{1 / p}, \quad \lambda>0,
$$

where $A$ is any measurable subset of $\mathbb{T}$ and $\chi_{A}$ denotes its characteristic function. From this, using arguments close to Yano's extrapolation theorem (see [13]) he went on and proved the norm estimate

2000 Mathematics Subject Classification: 42A20, 42B25.

Research partially supported by EC Grant via the network "Harmonic Analysis and Related Problems" and by the Göran Gustafsson Foundation. The second author was also supported by Grant BFM2001-0189. 


$$
\left\|S^{*} f\right\|_{L^{1}(\mathbb{T})} \leq C \int_{\mathbb{T}}|f(x)|\left(\log ^{+}|f(x)|\right)^{2} d x+C .
$$

This inequality, of course, implies the a.e. convergence of Fourier series of functions in $L(\log L)^{2}(\mathbb{T})$.

In [8], the first author used (1) in a more efficient way and showed that in fact the a.e. convergence is true for functions in $L \log L \log \log L(\mathbb{T})$. He also presented a more abstract approach valid for maximal operators associated to summability kernels satisfying an inequality like (1). In particular, his result was also applicable to the a.e. convergence of Walsh-Fourier series. (See also [11].)

In [1], N. Yu. Antonov strengthened the information contained in inequality (1) and proved that from it one can in fact obtain a.e. convergence of the Fourier series of functions in $L \log L \log \log \log L(\mathbb{T})$. In this paper we extend this result to other maximal convolution and non-convolution operators for which an inequality like (1) holds. Among other results, we prove the a.e. convergence of Walsh-Fourier series in the same class. Other applications include an improvement of estimates associated with the so called halo conjecture in differentiation of integrals.

Let us first summarize the approach presented in [1].

Lemma 1 (Antonov). Given $\varepsilon>0, a>0, N \in \mathbb{N}$ and a measurable function $f$ on $\mathbb{T}$ with $0 \leq f(x) \leq a$, there exists a set $F \subset \operatorname{supp} f$ such that

(i) $\left\|S_{N}^{*}\left(f-a \chi_{F}\right)\right\|_{\infty} \leq \varepsilon$,

(ii) $\int f=a|F|$.

This lemma is the key ingredient of the paper of Antonov. For its proof, he uses the fact that the Dirichlet kernels $D_{m}$ are in the class $C^{1}$ with

$$
\left\|D_{m}^{\prime}\right\|_{\infty} \leq m\left\|D_{m}\right\|_{\infty} \sim m^{2}
$$

(Bernstein's inequality).

An iteration of Lemma 1 then gives the following.

Lemma 2 (Antonov). Let $\psi:[0, \infty) \rightarrow[0, \infty)$ be a non-decreasing function such that $\psi\left(u^{2}\right) \leq C \psi(u)$ for $u \geq 4$. Define $\phi(u)=u \psi(u)$. Given $\varepsilon>0$, $N \in \mathbb{N}$ and $G \in \phi(L)$ (this simply means that $\int \phi(|G(x)|) d x<\infty$ ) with $G(x) \geq 4$ or $G(x)=0$ otherwise, there exists a sequence $\left\{F_{k}\right\}$ of disjoint sets such that the function

satisfies

$$
g(x)=\sum_{k \geq 1} 2^{2^{k}} \chi_{F_{k}}
$$

(i) $\left\|S_{N}^{*}(G-g)\right\|_{\infty}<\varepsilon$,

(ii) $\int \phi(g) \leq 4 \int \phi(G)$. 
For the conclusion on the a.e. convergence, we have not used estimate (1) so far. This is done in the following lemma.

Lemma 3. Assume that $M$ is a positive sublinear operator acting on simple functions of a measure space $(X, d \mu)$ and satisfying the condition

$$
\mu\left(\left\{x \in X: M \chi_{A}(x)>\lambda\right\}\right)^{1 / p} \leq\left(\frac{C}{p-1}\right)^{m} \frac{|A|^{1 / p}}{\lambda}
$$

for some $m \geq 0$ and for all $1<p \leq 2$, with $C$ independent of $p, \lambda>0$ and of the measurable set $A \subset X$ (that is, $M$ is of restricted weak type $p$ with a constant which grows like $(p-1)^{-m}$ as $\left.p \rightarrow 1^{+}\right)$. Let $\left\{a_{k}\right\}_{k}$ be an increasing sequence of positive numbers, with $a_{k} \geq 2$. Then, given a subset $X_{0} \subset X$ of finite measure and $\varepsilon>0$ small there is a positive, finite constant $C_{\varepsilon}$ such that for any simple function of the form

$$
g=\sum_{k=1}^{L} a_{k} \chi_{F_{k}}
$$

one has

$$
\mu\left(\left\{x \in X_{0}: M g(x)>1\right\}\right) \leq C_{\varepsilon} \sum_{k=1}^{L} a_{k}\left(\log (k+2)^{2} a_{k}\right)^{m} \log (2+k)\left|F_{k}\right|+\varepsilon .
$$

$\left(C_{\varepsilon}\right.$ might also depend on the value of $\mu\left(X_{0}\right)$.)

In the statement of the lemma and in what follows, we write $|A|$ for the $\mu$-measure of a measurable set $A$. As we will see, the assumption that $g$ is given by a finite sum may be removed if we know in advance that $M$ has the sublinearity property $M\left(\sum_{k} a_{k} \chi_{A_{k}}\right) \leq \sum_{k} a_{k} M\left(\chi_{A_{k}}\right)$ for sums of infinitely many terms, whenever $\sum_{k} a_{k} \chi_{A_{k}}$ is in the domain of definition of $M$.

Application of these results is then contained in the following theorem.

THEOREM 4. Let $\phi(u)=u\left(1+\log ^{+} u\right)\left(1+\log ^{+} \log ^{+} \log ^{+} u\right)$, where $\log ^{+} u$ $=\log u$ if $u \geq 1$ and $\log ^{+} u=0$ otherwise. Then for all $\varepsilon>0$ there is a constant $C_{\varepsilon}$ such that

$$
\left|\left\{x \in \mathbb{T}: S^{*} f(x)>\lambda\right\}\right| \leq C_{\varepsilon} \int \phi(|f| / \lambda)+\varepsilon .
$$

Lemma 3 for $m=1$ and Theorem 4 are of course implicit in the work of Antonov, although they are not stated in such explicit form. For that reason we briefly sketch their proofs.

Proof of Lemma 3. We start with the observation that if for a given $\lambda$ with $0<\lambda<1 / 2$ we take $p=1+(1+\log 1 / \lambda)^{-1}$ then $(2)$ gives

$$
\mu\left(\left\{x \in X: M \chi_{A}(x)>\lambda\right\}\right) \leq \frac{C}{\lambda}\left(\log \frac{1}{\lambda}\right)^{m}|A|, \quad 0<\lambda<1 / 2 .
$$


This is the only information from inequality (2) that we will use at this point.

Set $E_{k}=\left\{x: M \chi_{F_{k}}(x)>1 / a_{k}\right\}$ and $E=\bigcup E_{k}$. From (3) we get

$$
\mu(E) \leq C \sum_{k \geq 1} a_{k}\left(\log a_{k}\right)^{m}\left|F_{k}\right|
$$

Now,

$$
\mu\left(\left\{x \in X_{0}: M g(x)>1\right\}\right) \leq \mu(E)+\int_{X_{0}-E} M g d \mu,
$$

and

$$
\begin{aligned}
\int_{X_{0}-E} M g d \mu & \leq \sum_{k \geq 1} a_{k} \int_{X_{0}-E_{k}} M \chi_{F_{k}} d \mu \\
& \leq \sum_{k \geq 1} a_{k} \int_{0}^{1 / a_{k}} \mu\left(\left\{x \in X_{0}: M \chi_{F_{k}}(x)>\lambda\right\}\right) d \lambda \\
& \leq C \sum_{k \geq 1} a_{k} \int_{0}^{1 / a_{k}} \min \left(1, \frac{1}{\lambda}\left(\log \frac{1}{\lambda}\right)^{m}\left|F_{k}\right|\right) d \lambda,
\end{aligned}
$$

where we have used (3) again and the hypothesis that $\mu\left(X_{0}\right)$ is finite. Hence, for $0<\varepsilon<1 / 2$,

$$
\begin{aligned}
\int_{X_{0}-E} M g d \mu & \leq \sum_{k \geq 1} \frac{\varepsilon}{(k+2)^{2}}+\sum_{k \geq 1} a_{k} \int_{\varepsilon /(k+2)^{2} a_{k}}^{1 / a_{k}} \min \left(1, \frac{1}{\lambda}\left(\log \frac{1}{\lambda}\right)^{m}\left|F_{k}\right|\right) d \lambda \\
& \sim \varepsilon+\sum_{k \geq 1} a_{k}\left(\log \frac{(k+2)^{2} a_{k}}{\varepsilon}\right)^{m} \log \frac{(k+2)^{2}\left|F_{k}\right|}{\varepsilon} \\
& \leq \varepsilon+2\left(\log \frac{1}{\varepsilon}\right)^{m+1} \sum_{k=1}^{L} a_{k}\left(\log (k+2)^{2} a_{k}\right)^{m} \log (k+2)\left|F_{k}\right|,
\end{aligned}
$$

and the conclusion follows with $C_{\varepsilon} \sim(\log 1 / \varepsilon)^{m+1}$.

Proof of Theorem 4. We fix $\varepsilon>0$ and $f \in \phi(L)$ with $f \geq 0$. Given $\lambda>0$ write $f_{\lambda}(x)=f(x)$ if $f(x) \leq \lambda$ and $f_{\lambda}(x)=0$ otherwise. Set $f^{\lambda}=f-f_{\lambda}$ and

$$
G(x)=4 f^{\lambda}(x) / \lambda .
$$

Then

$$
\left|\left\{x: S^{*} f(x)>\lambda\right\}\right| \leq\left|\left\{x: S^{*} f_{\lambda}(x)>\lambda / 2\right\}\right|+\left|\left\{x: S^{*} G(x)>2\right\}\right| .
$$

For the first term we use the boundedness of $S^{*}$ on $L^{2}(\mathbb{T})$ to obtain

$$
\left|\left\{x: S^{*} f_{\lambda}(x)>\lambda / 2\right\}\right| \leq \frac{C}{\lambda^{2}} \int\left(f_{\lambda}\right)^{2} \leq C \int f / \lambda \leq C \int \phi(f / \lambda) .
$$


For the second term we use Lemma 2 with

$$
\psi(u)=\left(1+\log ^{+} u\right)\left(1+\log ^{+} \log ^{+} \log ^{+} u\right),
$$

$a_{k}=2^{2^{k}}, \varepsilon=1$ and $N$ arbitrary. We then find $g$ of the form $g(x)=$ $\sum_{k} a_{k} \chi_{F_{k}}$ with the $\left\{F_{k}\right\}$ disjoint and so that $\left\|S_{N}^{*}(G-g)\right\|_{\infty}<1$ and $\int \phi(g) \leq$ $4 \int \phi(G)$. Observe that $g$ may also depend on $N$. Using this, Lemma 3 and the fact that $a_{k} \geq k+2$ we obtain

$$
\begin{aligned}
\left|\left\{x: S_{N}^{*} G(x)>2\right\}\right| & \leq\left|\left\{x: S_{N}^{*} g(x)>1\right\}\right| \\
& \leq C_{\varepsilon} \sum a_{k} \log a_{k} \log (k+2)\left|F_{k}\right|+\varepsilon \\
& \sim C_{\varepsilon} \int \phi(g)+\varepsilon \leq C_{\varepsilon} \int \phi(G)+\varepsilon \leq C_{\varepsilon}^{\prime} \int \phi(f / \lambda)+\varepsilon .
\end{aligned}
$$

Since the last term does not depend on $N$ we conclude, by the monotonicity of the sequence $\left\{S_{N}^{*}\right\}_{N}$, that

$$
\left|\left\{x: S^{*} G(x)>2\right\}\right| \leq C_{\varepsilon}^{\prime} \int \phi(f / \lambda)+\varepsilon,
$$

and the theorem follows.

2. A general approximation principle. As we have mentioned before, Antonov uses the fact that the convolution kernels defining $S^{*}$, in this case the Dirichlet kernels, are smooth. For the applications that we have in mind this is perhaps a strong condition. Our first remark in this regard is that one can approximate a given function in the spirit of Lemmas 1 and 2 but with no smoothness assumption at all on the kernels. This is better described in the following result.

Lemma 5. Given a sequence $\left\{K_{j}\right\}_{j \geq 0} \subset L^{1}\left(\mathbb{R}^{n}\right)$ of integrable functions we define for $N=0,1,2, \ldots$ the maximal operators

$$
K_{N}^{*} f(x)=\sup _{0 \leq j \leq N}\left|K_{j} * f(x)\right| .
$$

Then, given $\varepsilon>0, N$, a positive function $H \in L^{1}\left(\mathbb{R}^{n}\right)$ and an increasing sequence $\left\{a_{k}\right\}_{k \geq 1}$ of positive numbers with $\lim a_{k}=\infty$, there exists a simple function $h$ of the form

$$
h=\sum_{k=1}^{k_{0}} a_{k} \chi_{F_{k}},
$$

for some $k_{0}$, so that

(i) $F_{k} \subset G_{k}:=\left\{x: a_{k-1}<H(x) \leq a_{k}\right\}, k=1,2, \ldots\left(a_{0}=0\right)$, with $\int_{G_{k}} H(x) d x=a_{k}\left|F_{k}\right|$,

(ii) $\int K_{N}^{*}(H-h)(x) d x \leq \varepsilon$. 
Proof. Given $\beta$, we write as before $H^{\beta}(x)=H(x)$ whenever $H(x) \geq \beta$ and $H^{\beta}(x)=0$ otherwise. Since

$$
\int\left|K_{N}^{*} H^{\beta}(x)\right| d x \leq \sum_{j=0}^{N} \int\left|K_{j} * H^{\beta}(x)\right| d x
$$

tends to zero as $\beta$ tends to infinity by the dominated convergence theorem, we may assume with no loss of generality that $H(x)$ is bounded, say $H(x)$ $\leq a_{k_{0}}$ for all $x$.

Consider a grid $\left\{Q_{i}\right\}_{i}$ of cubes covering $\mathbb{R}^{n}$, with disjoint interiors, whose diagonals have all length $\delta$, for some value of $\delta>0$ to be chosen later. Clearly,

$$
\int_{Q_{i} \cap G_{k}} H(x) d x \leq a_{k}\left|Q_{i} \cap G_{k}\right|,
$$

and so there exists a set $F_{k}^{i} \subset Q_{i} \cap G_{k}$ such that

$$
\int_{Q_{i} \cap G_{k}} H(x) d x=a_{k}\left|F_{k}^{i}\right| .
$$

Define $F_{k}=\bigcup_{i} F_{k}^{i}$ and $h(x)=\sum_{k=1}^{k_{0}} a_{k} \chi_{F_{k}}$. Thus, $h$ is of the form stated in the lemma and moreover,

$$
\begin{aligned}
\int K_{N}^{*}(H-h)(x) d x \leq \sum_{l=0}^{N} \int\left|K_{l} *(H-h)(x)\right| d x \\
\quad \leq \sum_{l=0}^{N} \sum_{k=1}^{k_{0}} \sum_{i} \int_{\mathbb{R}^{n}}\left|\int_{Q_{i} \cap G_{k}} K_{l}(x-y)\left(H(y)-a_{k} \chi_{F_{k}^{i}}(y)\right) d y\right| d x .
\end{aligned}
$$

Observe that, by construction,

$$
\int_{Q_{i} \cap G_{k}}\left(H(y)-a_{k} \chi_{F_{k}^{i}}(y)\right) d y=0
$$

and therefore if we write $y_{i}$ for the center of the cube $Q_{i}$, we obtain

$$
\begin{aligned}
& \int K_{N}^{*}(H-h)(x) d x \\
& \leq \sum_{l=0}^{N} \sum_{k=1}^{k_{0}} \sum_{i} \int_{\mathbb{R}^{n}}\left|\int_{Q_{i} \cap G_{k}}\left(K_{l}(x-y)-K_{l}\left(x-y_{i}\right)\right)\left(H(y)-a_{k} \chi_{F_{k}^{i}}(y)\right) d y\right| d x \\
& \leq \sum_{l=0}^{N} \sum_{k=1}^{k_{0}} \sum_{i} \int_{Q_{i} \cap G_{k}}\left(H(y)+a_{k} \chi_{F_{k}^{i}}(y)\right)\left(\int_{\mathbb{R}^{n}}\left|K_{l}(x-y)-K_{l}\left(x-y_{i}\right)\right| d x\right) d y .
\end{aligned}
$$

Well known properties of the translation operator tell us that if we choose 
$\delta$ sufficiently small, then

$$
\int_{\mathbb{R}^{n}}\left|K_{l}(x-z)-K_{l}(x)\right| d x \leq \varepsilon_{0}, \quad l=1, \ldots, N,
$$

whenever $|z|<\delta$, with $\varepsilon_{0}$ to be determined. Hence, for such $\delta$,

$$
\int K_{N}^{*}(H-h)(x) d x \leq \sum_{l=0}^{N} \sum_{k=1}^{k_{0}} \sum_{i} 2 \varepsilon_{0} \int_{Q_{i} \cap G_{k}} H(y) d y=2 \varepsilon_{0}(N+1) \int H(y) d y .
$$

Choosing

$$
\varepsilon_{0}=\frac{\varepsilon}{4(N+1)\left(1+\|H\|_{1}\right)}
$$

completes the proof.

Part (i) in the conclusion of Lemma 5 produces the following nice connection between the norms of $h$ and $H$ in certain $\phi(L)$-spaces.

LEMma 6. In the hypotheses of Lemma 5 and with the same notation, if $\widetilde{\phi}(u)=u \widetilde{\psi}(u)$ with $\widetilde{\psi}(u)$ increasing in $[0, \infty), \widetilde{\psi}(0)>0$ and satisfying the condition

$$
\widetilde{\psi}\left(a_{k}\right) \leq C_{1} \widetilde{\psi}\left(a_{k-1}\right), \quad k=1,2, \ldots,
$$

for the given sequence $\left\{a_{k}\right\}_{k}$, then

$$
\int \widetilde{\phi}(h) \leq C_{1} \int \widetilde{\phi}(H) .
$$

Proof. We observe, from the construction of the sequence $\left\{F_{k}\right\}_{k}$, that

$$
\begin{aligned}
\int \widetilde{\phi}(h) & =\sum_{k} \widetilde{\phi}\left(a_{k}\right)\left|F_{k}\right|=\sum_{k} a_{k}\left|F_{k}\right| \widetilde{\psi}\left(a_{k}\right) \\
& =\sum_{k} \int_{G_{k}} H(x) d x \widetilde{\psi}\left(a_{k}\right) .
\end{aligned}
$$

Now, our hypothesis (4) and the fact that $\widetilde{\psi}$ is increasing gives, for every $x \in G_{k}$,

$$
\widetilde{\psi}\left(a_{k}\right) \leq C_{1} \widetilde{\psi}\left(a_{k-1}\right) \leq C_{1} \widetilde{\psi}(H(x)) .
$$

Hence,

$$
\int \widetilde{\phi}(h) \leq C_{1} \sum_{k} \int_{G_{k}} H(x) \widetilde{\psi}(H(x)) d x=C_{1} \int \widetilde{\phi}(H)
$$

3. Boundedness of the maximal operator. In this section we present the generalization of Antonov's work announced in the introduction. Our setting will be $\mathbb{R}^{n}$ and a sequence $\left\{K_{j}\right\}_{j}$ of kernels in $L^{1}\left(\mathbb{R}^{n}\right)$. We define the associated maximal operator

$$
K^{*} f(x)=\sup _{j}\left|K_{j} * f(x)\right|,
$$


and we assume that $K^{*}$ has the following behavior on characteristic functions of sets:

$$
\left|\left\{x: K^{*} \chi_{A}(x)>\lambda\right\}\right|^{1 / p} \leq\left(\frac{C}{p-1}\right)^{m} \frac{|A|^{1 / p}}{\lambda}
$$

for some $m \geq 0$ and for all $1<p \leq 2$, with $C$ independent of $p, \lambda>0$ and of the measurable set $A$. Set $\psi_{m}(u)=\left(1+\log ^{+} u\right)^{m}, \phi_{m}(u)=u \psi_{m}(u)$ and $\widetilde{\phi}_{m}(u)=\phi_{m}(u)\left(1+\log ^{+} \log ^{+} \log ^{+} u\right)$.

THEOREM 7. With the above notation and assuming that $K^{*}$ satisfies (5), given $\varepsilon>0$ and $R>0$ there exists a constant $C_{\varepsilon}$, which may also depend on $R$, so that

$$
\left|\left\{x \in \mathbb{R}^{n}:|x| \leq R, K^{*} f(x)>\lambda\right\}\right| \leq C_{\varepsilon} \int \widetilde{\phi}_{m}(|f| / \lambda)+\varepsilon .
$$

We say that the sequence $\left\{K_{j}\right\}_{j}$ is a pointwise summability system for smooth functions if the limit

$$
\lim _{j \rightarrow \infty} K_{j} * f(x)
$$

exists for every $f$ in the Schwartz class $\mathcal{S}$, a.e. $x$.

COROLlary 8. If $\left\{K_{j}\right\}_{j}$ is a pointwise summability system for smooth functions and its associated maximal operator satisfies condition (5), then the limit

$$
\lim _{j \rightarrow \infty} K_{j} * f(x)
$$

exists a.e. $x$ for all $f \in \widetilde{\phi}_{m}(L)$.

Proof of Theorem 7. The argument is similar to that in the proof of Theorem 4 but with the modifications given by Lemmas 5 and 6 . First of all we observe that condition (5) says that $K^{*}$ is of restricted weak type $p$ for $1<p \leq 2$. Therefore, well known results in interpolation theory (see [12]) tell us that it is also of strong type $p$ for $1<p<2$. That is,

$$
\left\|K^{*} f\right\|_{p} \leq C_{p}\|f\|_{p}
$$

for some $C_{p}$. Fix $R>0$. In order to prove (6), it suffices to consider $f \geq 0$ and, by homogeneity, we may as well take simply $\lambda=2$. Write as usual

$$
f=f_{1}+f^{1}
$$

where $f_{1}(x)=f(x)$ if $f(x) \leq 1$ and $f_{1}(x)=0$ otherwise. Then

$$
\begin{aligned}
\left|\left\{x:|x| \leq R, K^{*} f(x)>2\right\}\right| \leq \mid & \left\{x: K^{*} f_{1}(x)>1\right\} \mid \\
& +\left|\left\{x:|x| \leq R, K^{*} f^{1}(x)>1\right\}\right| .
\end{aligned}
$$

For the first term we use the boundedness of $K^{*}$ on, say, $L^{3 / 2}$ to obtain

$$
\left|\left\{x: K^{*} f_{1}(x)>1\right\}\right| \leq \int\left(K^{*} f_{1}\right)^{3 / 2} \leq C \int\left(f_{1}\right)^{3 / 2} \leq C \int f \leq C \int \widetilde{\phi}_{m}(f) .
$$


For the second term, given $\varepsilon>0$ and $N$ fixed we use Lemma 5 with $H=f^{1}$ and $a_{k}=e^{e^{k}}$. We then find $g$ of the form $g(x)=\sum_{k} a_{k} \chi_{F_{k}}$ with the $\left\{F_{k}\right\}$ disjoint and such that

$$
\left\|K_{N}^{*}\left(f^{1}-g\right)\right\|_{1}<\varepsilon / 2 .
$$

Observe that

$$
\psi_{m}\left(a_{k}\right) \log (k+2) \leq C \psi_{m}\left(a_{k-1}\right) \log (k+1),
$$

and since $\log k=\log \log \log a_{k}$ we see that condition (4) in Lemma 6 is satisfied with

$$
\widetilde{\psi}(u)=\psi_{m}(u)\left(1+\log ^{+} \log ^{+} \log ^{+} u\right) .
$$

Hence

$$
\int \widetilde{\phi}_{m}(g) \leq C \int \widetilde{\phi}_{m}\left(f^{1}\right) \leq C \int \widetilde{\phi}_{m}(f) .
$$

Finally, (9), Lemma 3 and (10) give

$$
\begin{aligned}
\mid\{x: \mid & \left.|x| \leq R, K^{*} f^{1}(x)>1\right\} \mid \\
& \leq\left|\left\{x: K^{*}\left(f^{1}-g\right)(x)>1 / 2\right\}\right|+\left|\left\{x:|x| \leq R, K^{*} g(x)>1 / 2\right\}\right| \\
& \leq \varepsilon+C_{\varepsilon} \sum a_{k} \psi_{m}\left(a_{k}\right) \log (k+2)\left|F_{k}\right| \\
& \leq \varepsilon+C_{\varepsilon} \int \widetilde{\phi}_{m}(g) \leq \varepsilon+C_{\varepsilon} \int \widetilde{\phi}_{m}(f) .
\end{aligned}
$$

This finishes the proof of Theorem 7 .

Proof of Corollary 8. The proof is standard. We want to show that

$$
\limsup _{j \rightarrow \infty} K_{j} * f(x)=\liminf _{j \rightarrow \infty} K_{j} * f(x) \quad \text { a.e. }
$$

To do that, it suffices to prove that for every $R>0$ and $\lambda>0$, if we define

$$
m_{R}=\left|\left\{x:|x| \leq R, \limsup _{j \rightarrow \infty} K_{j} * f(x)-\liminf _{j \rightarrow \infty} K_{j} * f(x)>\lambda\right\}\right|
$$

then we have $m_{R}=0$. Now, if we take a smooth function $g \in \mathcal{S}$, our hypothesis on $\left\{K_{j}\right\}_{j}$ gives

$$
m_{R}=\left|\left\{x:|x| \leq R, \limsup _{j \rightarrow \infty} K_{j} *(f-g)(x)-\liminf _{j \rightarrow \infty} K_{j} *(f-g)(x)>\lambda\right\}\right| .
$$

Moreover, from Theorem 7 , given $\varepsilon>0$, there exists $C_{\varepsilon}$ such that

$$
m_{R} \leq\left|\left\{x:|x| \leq R, 2 K^{*}(f-g)(x)>\lambda\right\}\right| \leq \varepsilon+C_{\varepsilon} \int \widetilde{\phi}\left(2 \frac{f-g}{\lambda}\right) .
$$

Since we can make the second term in the last inequality as small as we want for appropriate $g \in \mathcal{S}$, the corollary follows. 
Remark. As the reader can easily see, Theorem 7 and Corollary 8 work for periodic kernels $\left\{K_{j}\right\}_{j}$. In that case, estimate (6) concerns the level set restricted to the corresponding torus $\mathbb{T}^{n}=[0,1)^{n}$.

\section{Some examples}

4.1. A.e. convergence of Fourier series. In dimension $n=1$, this is of course the example which motivated Antonov's result. In higher dimensions, if $K_{j} * f$ denotes the Fourier partial sum of the function $f$ in $\mathbb{T}^{n}$ associated to the frequencies $k=\left(k_{1}, \ldots, k_{n}\right) \in \mathbb{Z}^{n}$ with $\left|k_{i}\right| \leq j, i=1, \ldots, n$, then the first author [9] showed that the associated maximal operator, $K^{*}$, satisfies (essentially) the pointwise inequality

$$
K^{*} f(x) \leq \sum_{i=1}^{n} T_{i}\left(L_{i} f\right)\left(x_{1}+\ldots+x_{n}\right)+\pi^{n}|f(x)|,
$$

where $T_{i}$ is the 1-dimensional maximal Carleson operator acting on the variable $i$ and $L_{i} f$ is the composition of $n-1$ Hilbert transforms, each acting on one of the variables $1, \ldots, i-1, i+1, \ldots, n$ of the function

$$
g_{i}\left(u_{1}, \ldots, u_{n}\right)=f\left(u_{1}, \ldots, u_{i-1}, u_{i}-\sum_{j \neq i} u_{j}, u_{i+1}, \ldots, u_{n}\right) .
$$

For $m>0$ define $\widetilde{\phi}_{m}(u)=u\left(1+\log ^{+} u\right)^{m}\left(1+\log ^{+} \log ^{+} \log ^{+} u\right)$ as in Section 3. From Theorem 4, we know that given $\varepsilon>0$ there exists $C_{\varepsilon}$ such that for each $i=1, \ldots, n$,

$$
\left|\left\{x \in \mathbb{T}^{n}: T_{i} F(x)>\lambda\right\}\right| \leq C_{\varepsilon} \int_{\mathbb{T}^{n}} \widetilde{\phi}_{1}(|F(x)| / \lambda) d x+\varepsilon .
$$

We also know (see [13]) that if $H$ denotes the ordinary Hilbert transform then for $m=1,2, \ldots$ and $\varepsilon>0$ there exists $C_{\varepsilon}$ such that

$$
\int_{\mathbb{T}} \widetilde{\phi}_{m}(|H g(x)|) d x \leq C_{\varepsilon} \int_{\mathbb{T}} \widetilde{\phi}_{m+1}(|g(x)|) d x+\varepsilon .
$$

So, we conclude that

$$
\left|\left\{x \in \mathbb{T}^{n}: K^{*} f(x)>\lambda\right\}\right| \leq C_{\varepsilon} \int_{\mathbb{T}^{n}} \widetilde{\phi}_{n}(|f(x)| / \lambda) d x+\varepsilon .
$$

Corollary 8 then gives the a.e. convergence of multiple Fourier series under summation over cubes, for every function in the class $L(\log L)^{n} \log \log \log L$. (This was also announced in [1].)

4.2. Differentiation of integrals and the halo conjecture. This is perhaps an area where our results fit better. Let us begin with some definitions and notation. A collection $\mathcal{B}$ of bounded measurable subsets of $\mathbb{R}^{n}$ is called a 
differentiation basis if we can write

$$
\mathcal{B}=\bigcup_{x \in \mathbb{R}^{n}} \mathcal{B}(x),
$$

so that for all $x \in \mathbb{R}^{n}$ and $A \in \mathcal{B}(x)$ we have $x \in A$ and we can index $\mathcal{B}(x)=\left\{A_{\alpha}^{x}\right\}_{\alpha \in(0,1)}$ in such a way that

$$
\lim _{\alpha \rightarrow 0^{+}} \operatorname{diam}\left(A_{\alpha}^{x}\right)=0 .
$$

The theory of differentiation of integrals studies the problem of determining those classes of functions $\phi(L)$ for which one has the extension of the Lebesgue differentiation theorem, that is,

$$
\lim _{\alpha \rightarrow 0^{+}} \frac{1}{\left|A_{\alpha}^{x}\right|} \int_{A_{\alpha}^{x}} f(y) d y=f(x) \quad \text { a.e., } \forall f \in \phi(L) .
$$

Whenever (12) holds we say that $\mathcal{B}$ differentiates $\phi(L)$.

As usual this problem is directly related to proving the boundedness of the maximal operator

$$
M_{\mathcal{B}} f(x)=\sup _{x \in A \in \mathcal{B}(x)} \frac{1}{|A|}\left|\int_{A} f(y) d y\right| .
$$

$\mathcal{B}$ is called a Busemann-Feller basis if, in addition, it is invariant under translations and dilations. The so called halo conjecture states that if $\mathcal{B}$ is a Busemann-Feller basis and there exists a constant $C$ so that

$$
\left|\left\{x: M_{\mathcal{B}} \chi_{D}(x)>1 / u\right\}\right| \leq C \phi(u)|D|
$$

for every measurable subset $D \subset \mathbb{R}^{n}$, then $\mathcal{B}$ differentiates $\phi(L)$. Here one assumes that $\phi$ is increasing and that $\phi(u) \geq c u$.

The conjecture has been proved so far only in the special case $\phi(u) \sim u$ (see the monograph [6] by Miguel de Guzmán for a complete description of the theory of differentiation of integrals and of this particular problem).

In [10], the second author found partial results in the general case by showing that if (14) holds then $\mathcal{B}$ differentiates $\widetilde{\phi}(L)$ for $\widetilde{\phi}(u)=\phi(u)(1+$ $\left.\log ^{+} \log ^{+} u\right)$. We can now improve this using the tools developed in the previous sections. As in [10] we only assume that $\mathcal{B}$ is invariant under translations, that is,

$$
A \in \mathcal{B}(x) \Leftrightarrow A-x \in \mathcal{B}(0), \quad \forall x \in \mathbb{R}^{n} .
$$

Thus, if $\mathcal{B}(0)=\left\{A_{\alpha}\right\}_{\alpha \in(0,1)}$ and $K_{\alpha}=\left|A_{\alpha}\right|^{-1} \chi_{A_{\alpha}}$, we can write the maximal operator in (13) as

$$
M_{\mathcal{B}} f(x)=\sup _{0<\alpha<1}\left|K_{\alpha} * f(x)\right| .
$$

Let us consider now a function $\phi(u)=u \psi(u)$ where $\psi(u)$ is a nondecreasing function on $[0, \infty)$ with $\psi(0)>0$. Assume that $E:[0, \infty) \rightarrow$ 
$[0, \infty)$ is continuous, strictly increasing, unbounded, and there exists a constant $C$ so that if we define $a_{k}=E(k), k=1,2, \ldots$, then

$$
\psi\left(a_{k}\right) \leq C \psi\left(a_{k-1}\right), \quad \psi\left(\frac{(k+1)^{2} a_{k}}{\varepsilon}\right) \leq C_{\varepsilon} \psi\left(a_{k}\right) .
$$

Define also the function

$$
\widetilde{\phi}(u)=\phi(u)\left(1+\log ^{+} E^{-1}(u)\right), \quad u \geq 0 .
$$

( $E^{-1}$ may not be defined for small values of $u$. By convention we let $E^{-1}(u)$ $=0$ if $0 \leq u \leq E(0)$.) Then we have

THEOREM 9. With the previous notation and definitions, if $\mathcal{B}$ is a differentiation basis invariant under translations and the associated maximal operator satisfies (14) for $\phi(u)$, then $\mathcal{B}$ differentiates $\widetilde{\phi}(L)$ where $\widetilde{\phi}$ is defined in (16).

Before we proceed, some examples are in order. Take $\phi(u)=\phi_{m}(u)=$ $u\left(1+\log ^{+} u\right)^{m}$. (Observe that in this case inequality (14) is the same as (3) with $M$ replaced with $M_{\mathcal{B}}$.) Our choice of the function $E$ for (15) to hold is

$$
E(u)=e^{e^{u}}
$$

and so $E^{-1}(u)=\log \log u(u>e)$. Theorem 9 then tells us that $\mathcal{B}$ differentiates $L\left(\log ^{+} L\right)^{m}\left(\log ^{+} \log ^{+} \log ^{+} L\right)$.

Also, for $\phi(u)=u\left(1+\log ^{+} \ldots{ }^{\alpha} \ldots \log ^{+} u\right)^{m}=: u\left(\log ^{(\alpha)}(u)\right)^{m}$, for some $\alpha \in \mathbb{N}$, we can take $E(u)=\exp \left(\ldots^{\alpha+1} \ldots \exp (u)\right)$ and (15) holds. Since $E^{-1}(u) \sim \log { }^{(\alpha+1)}(u)$ for $u$ large, Theorem 9 says that if $M_{\mathcal{B}}$ satisfies inequality (14) for this $\phi$ then $\mathcal{B}$ differentiates $\widetilde{\phi}(L)$ with $\widetilde{\phi}(u)=\phi(u) \log ^{(\alpha+2)}(u)$. The best known result to date in this case was that $\mathcal{B}$ differentiated $\widetilde{\phi}(L)$ with $\widetilde{\phi}(u)=\phi(u) \log ^{(2)}(u)$.

Proof of Theorem 9. As in Section 3, our goal is an estimate of the sort

$$
\left|\left\{x:|x| \leq R, M_{\mathcal{B}} f(x)>\lambda\right\}\right| \leq C_{\varepsilon} \int \widetilde{\phi}(|f| / \lambda)+\varepsilon .
$$

Once this is done, the rest is easy. For if (11) holds then the limit (12) holds everywhere for continuous functions. This says in particular that the family of kernels

$$
K_{\alpha}=\left|A_{\alpha}\right|^{-1} \chi_{A_{\alpha}}, \quad 0<\alpha<1,
$$

is a pointwise summability system for smooth functions, and so the arguments of Corollary 8 apply.

Consider an arbitrary finite subset $\Gamma \subset(0,1)$ and define

$$
M_{\Gamma} f(x)=\sup _{\alpha \in \Gamma}\left|K_{\alpha} * f(x)\right| .
$$

If we can prove (17) with $M_{\Gamma}$ replacing $M_{\mathcal{B}}$, with a constant $C_{\varepsilon}$ independent of $\Gamma$, it is clear that the same will hold, by monotonicity, for $M_{\mathcal{B}}$. By 
homogeneity, we can assume that $\lambda=2$ and that $f(x) \geq 4$ in its support. Invoking Lemma 5 , given $\varepsilon>0$ we find a simple function of the form

$$
g=\sum_{k=1}^{k_{0}} a_{k} \chi_{F_{k}}
$$

with

$$
\begin{gathered}
F_{k} \subset G_{k}=\left\{x: a_{k-1}<f(x) \leq a_{k}\right\}, \quad k=1,2, \ldots \quad\left(a_{0}=0\right), \\
\int_{G_{k}} f=a_{k}\left|F_{k}\right|, \quad \int M_{\Gamma}(f-g)(x) d x \leq \varepsilon .
\end{gathered}
$$

Thus, we only need to estimate

$$
\left|\left\{x:|x| \leq R, M_{\Gamma}\left(\sum_{k=1}^{k_{0}} a_{k} \chi_{F_{k}}\right)(x)>1\right\}\right| .
$$

But this is done, basically, in the proof of Lemma 3. Here we replace (3) with (14), that is,

$$
\left|\left\{x: M_{\mathcal{B}} \chi_{A}(x)>\lambda\right\}\right| \leq C_{0} \phi\left(\frac{1}{\lambda}\right)|A|=C_{0} \frac{1}{\lambda} \psi\left(\frac{1}{\lambda}\right)|A|,
$$

and obtain, following the same steps,

$$
\begin{aligned}
\mid\{x:|x| & \left.\leq R, M_{\Gamma}\left(\sum_{k=1}^{k_{0}} a_{k} \chi_{F_{k}}\right)(x)>1\right\} \mid \\
& \leq C_{0} \sum_{k} \phi\left(a_{k}\right)\left|F_{k}\right|+\varepsilon+C \sum_{k} a_{k} \int_{\varepsilon /(k+2)^{2} a_{k}}^{1 / a_{k}} \frac{1}{\lambda} \psi\left(\frac{1}{\lambda}\right)\left|F_{k}\right| d \lambda \\
& \leq \varepsilon+C \sum_{k} a_{k}\left|F_{k}\right| \psi\left(\frac{(k+2)^{2} a_{k}}{\varepsilon}\right) \log \frac{(k+2)^{2}}{\varepsilon} \\
& \leq \varepsilon+C_{\varepsilon} \sum_{k} a_{k}\left|F_{k}\right| \psi\left(a_{k}\right) \log (k+2) \\
& =\varepsilon+C_{\varepsilon} \sum_{k} \int_{G_{k}} f(x) \psi\left(a_{k}\right) \log (k+2) d x .
\end{aligned}
$$

Since, by hypothesis, $\psi\left(a_{k}\right) \sim \psi\left(a_{k-1}\right), \log (k+2) \sim 1+\log \left(E^{-1}\left(a_{k-1}\right)\right)$ and $f(x) \geq a_{k-1}$ for all $x \in G_{k}$, we obtain

$$
\begin{aligned}
\mid\{x:|x| & \left.\leq R, M_{\Gamma}\left(\sum_{k=1}^{k_{0}} a_{k} \chi_{F_{k}}\right)(x)>\lambda\right\} \mid \\
& =\varepsilon+C_{\varepsilon} \int f(x) \psi(f(x))\left(1+\log \left(E^{-1} f(x)\right)\right) d x \sim \varepsilon+C_{\varepsilon} \int \widetilde{\phi}(f(x)) d x,
\end{aligned}
$$

and the theorem follows. 
4.3. Restricted weak type 1 implies weak type 1 for a maximal convolution operator

THEOREM 10. Let $\left\{K_{j}\right\}_{j}$ be a sequence of integrable kernels in $\mathbb{R}^{n}$ and assume that the maximal operator

$$
K^{*} f(x)=\sup _{j}\left|K_{j} * f(x)\right|
$$

satisfies the inequality

$$
\left|\left\{x: K^{*} \chi_{A}(x)>\lambda\right\}\right| \leq \frac{C}{\lambda}|A|
$$

for all $\lambda>0$ and every measurable set $A$. Then

$$
\left|\left\{x: K^{*} f(x)>\lambda\right\}\right| \leq \frac{C}{\lambda} \int|f| .
$$

The theorem tells us that for an operator of this sort, the restricted weak type 1 boundedness (18) is indeed equivalent to its weak type 1 boundedness (19). This is reminiscent of what we have said before about the halo conjecture when the function $\phi(u)$ of inequality (14) is $\phi(u) \sim u$. The original result is due to Moon (see [3]). However we have not been able to find in the literature a proof more direct than the one presented here.

Proof of Theorem 10. As usual, we only need to prove (19) for the truncated maximal operator

$$
K_{N}^{*} f(x)=\sup _{j \leq N}\left|K_{j} * f(x)\right|,
$$

with constant $C$ independent of $N$. We may also take $f$ positive and bounded, say $0 \leq f(x) \leq \beta$. Now, the initial argument in the proof of Lemma 5 (with $a_{k_{0}}=a_{1}=\beta$ ) says that given $\varepsilon>0$ there exists a set $F$ so that

$$
\int f(x) d x=\beta|F|, \quad \int K_{N}^{*}\left(f-\beta \chi_{F}\right)(x) d x \leq \varepsilon .
$$

Therefore

$$
\begin{aligned}
\left|\left\{x: K_{N}^{*} f(x)>\lambda\right\}\right| & \leq \frac{2}{\lambda} \int K_{N}^{*}\left(f-\beta \chi_{F}\right)(x) d x+\left|\left\{x: K_{N}^{*} \beta \chi_{F}(x)>\lambda / 2\right\}\right| \\
& \leq \frac{2}{\lambda} \varepsilon+C \frac{\beta}{\lambda}|F|=\frac{2}{\lambda} \varepsilon+C \int \frac{f(x)}{\lambda} d x,
\end{aligned}
$$

and since $\varepsilon$ is arbitrary, the conclusion follows.

5. Non-convolution case. The main idea behind the results presented in this work, or in the work of Antonov [1], is that we can approximate in the $L^{1}$-norm (or in the $L^{\infty}$-norm in the case of Antonov) the action of the truncated maximal operators $K_{N}^{*}$ on certain classes of functions by simple functions for which we then have a nice weak type estimate. This approximation is possible due to the convolution structure of our operators 
and the continuity of the translation operator. The extension to the nonconvolution case is also possible, provided some additional assumptions are considered. This is presented in the following result.

Theorem 11. Let $X$ denote either the Euclidean space $\mathbb{R}^{n}$ or the $n$ dimensional torus $\mathbb{T}^{n}$. Let $\left\{K_{j}(x, y)\right\}_{j}$ be a sequence of measurable kernels defined on $X \times X$ satisfying the conditions

(i) $K_{j}(x, \cdot) \in L^{1}(X)$, a.e. $x$,

(ii) $K_{j}(\cdot, y) \in L^{1}(X)$, uniformly in $y$,

(iii) for all $\varepsilon>0$ and $j$, there exists $\delta>0$ and a grid $\left\{Q_{i}\right\}_{i}$ of disjoint cubes in $X$ whose diagonals have all length $\delta$, so that if $y_{1}, y_{2}$ belong to the same cube, one has

$$
\int_{X}\left|K_{j}\left(x, y_{1}\right)-K_{j}\left(x, y_{2}\right)\right| d x \leq \varepsilon .
$$

Then, if the maximal operator

$$
K^{*} f(x)=\sup _{j}\left|\int K_{j}(x, y) f(y) d y\right|
$$

satisfies estimate (5) for some $m \geq 0$ (restricted weak type $p$ with constant $(p-1)^{-m}$ as $\left.p \rightarrow 1^{+}\right)$then $K^{*}$ also satisfies (6) (weak type on $\widetilde{\phi}_{m}(L)$ ).

The proof follows step by step the same argument given in the proof of Theorem 7. Here, we only have to justify the existence of the function $g=\sum_{k} a_{k} \chi_{A_{k}}$ with $a_{k}=e^{e^{k}}$, for which the approximating estimate (9) holds. This, in turn, follows from a simple modification of the construction given in Lemma 5 , where the $L^{1}$-continuity of the translation operator is replaced by condition (iii) in the hypotheses. The details are left to the reader.

As in the case of convolution maximal kernels, the case $m=0$ (restricted weak type 1) of Theorem 11 can be improved.

THEOREM 12. Under the same hypotheses on the kernels $\left\{K_{j}(x, y)\right\}_{j}$ in Theorem 11, if $K^{*}$ is of restricted weak type 1 , then it is also of weak type 1.

The proof is the same as that of Theorem 10 with the modifications of Lemma 5 indicated above.

5.1. A.e. convergence of Walsh-Fourier series. An interesting application of Theorem 11 is given here.

THEOREM 13. The Walsh-Fourier series of functions in the class $L \log L \log \log \log L$ converge a.e. 
Proof. The $j$ th partial sum of the Walsh-Fourier series of a function $f$ is given by the operator

$$
\widetilde{S} f(x)=\int_{\mathbb{T}} K_{j}(x, y) f(y) d y,
$$

where

$$
K_{j}(x, y)=\sum_{l=0}^{j} \omega_{l}(x) \omega_{l}(y),
$$

and $\left\{\omega_{l}\right\}_{l}$ is the Walsh system. It is clear that $K_{j}$ satisfies conditions (i) and (ii) of Theorem 11 since, in fact, each $\omega_{l}$ is a bounded function. Now, estimate (5) for $m=1$ was proved by the first author in [8]. So, we only have to check the continuity condition (iii). It turns out that this follows from the following trivial observation: the Walsh function $\omega_{l}$ is constant on dyadic intervals of the form $\Delta_{i, N}=\left[(i-1) / 2^{N}, i / 2^{N}\right)$ if $2^{N} \geq 2 l$. Hence, $K_{j}(x, \cdot)$ is constant, for fixed $x$, on the intervals $\Delta_{i, N}$ if $N \geq j$. If we take $Q_{i}=\Delta_{i, N}, i=1, \ldots, N$, with $N \geq j$, and $y_{1}, y_{2} \in Q_{i}$ then

$$
K_{j}\left(x, y_{1}\right)-K_{j}\left(x, y_{2}\right)=0, \quad \forall x .
$$

This is of course stronger than condition (iii) and the theorem follows.

Observe that this result could not be obtained directly from the arguments in [1], since the kernels here are neither convolutors nor Lipschitz.

6. Further results and comments. There is a different approach to some of the results in this paper that we would like to present here and which is close in spirit to some recent results by Arias de Reina [2] (see [5] for yet another approach). For simplicity we will only consider the convolution case on $\mathbb{T}$. Let $\left\{K_{j}\right\}_{j}$ be a sequence of kernels in $L^{1}(\mathbb{T})$. Suppose that there exists a class of functions $B$, endowed with a homogeneous functional $\|\cdot\|_{B}$ (so that $\left.f \in B \Leftrightarrow\|f\|_{B}<\infty\right)$ and that the maximal operator $K^{*}$ sends $B$ boundedly into the space $L^{1, \infty}$; that is,

$$
\left|\left\{x: K^{*} f(x)>\lambda\right\}\right| \leq C_{0}\|f\|_{B} / \lambda .
$$

Then we can improve this estimate to a larger space defined as follows:

Definition. We say that $f \in L^{1}(\mathbb{T})$ is in the class $[B]=\mathcal{L}$ if there is a finite constant $C$ so that for all $\varepsilon>0$ and $N \in \mathbb{N}$ we can find $g \in B$ so that

$$
\begin{aligned}
\left\|K_{N}^{*}(f-g)\right\|_{L^{1}} & \leq \varepsilon, \\
\|g\|_{B} & \leq C .
\end{aligned}
$$

Observe that the class $\mathcal{L}$ depends on both $B$ and the sequence $\left\{K_{j}\right\}_{j}$ of kernels. 
We define $\|f\|_{\mathcal{L}}$ as the infimum of the above constants $C$; that is,

$$
\|f\|_{\mathcal{L}}=\sup _{\varepsilon>0} \sup _{N \in \mathbb{N}} \inf \left\{\|g\|_{B}:\left\|K_{N}^{*}(f-g)\right\|_{L^{1}} \leq \varepsilon\right\} .
$$

$\mathcal{L}$ and $\|\cdot\|_{\mathcal{L}}$ inherit the properties of $B$ and $\|\cdot\|_{B}$, respectively. In particular we have the following result.

THEOREM 14. If $K^{*}$ satisfies (20) with constant $C_{0}$, and $\mathcal{L}=[B]$ is as defined before, then

$$
\left|\left\{x: K^{*} f(x)>\lambda\right\}\right| \leq C_{0}\|f\|_{\mathcal{L}} / \lambda .
$$

Proof. This is a simple exercise. As usual, it suffices to prove (24) with $K^{*}$ replaced by $K_{N}^{*}$, for all $N$. Fix $\lambda$ and let $0<\eta<\lambda$. Given $\varepsilon$ we can take, by the definition of $\mathcal{L}$, a function $g \in B$ so that

$$
\left\|K_{N}^{*}(f-g)\right\|_{L^{1}} \leq \eta \varepsilon, \quad\|g\|_{B} \leq\|f\|_{\mathcal{L}}+\varepsilon .
$$

Then

$$
\begin{aligned}
\left|\left\{x: K_{N}^{*} f(x)>\lambda\right\}\right| & \leq\left|\left\{x: K_{N}^{*}(f-g)(x)>\eta\right\}\right|+\left|\left\{x: K_{N}^{*} g(x)>\lambda-\eta\right\}\right| \\
& \leq \varepsilon+C_{0} \frac{\|g\|_{B}}{\lambda-\eta} \leq \varepsilon+C_{0} \frac{\|f\|_{\mathcal{L}}+\varepsilon}{\lambda-\eta} .
\end{aligned}
$$

Since $\varepsilon$ and $\eta$ can be taken arbitrarily small, the result follows.

If $K^{*}$ satisfies estimate (5) for some $m \geq 0$ (restricted weak type $p$ with constant $(p-1)^{-m}$ as $\left.p \rightarrow 1^{+}\right)$then it was proven in [11] that $K^{*}$ satisfies (20) for a certain class $B_{m}^{*}$ of functions close to $L(\log L)^{m}$. Define

$$
\mathcal{L}_{m}=\left[B_{m}^{*}\right] .
$$

THEOREM 15. For $m \geq 0$, independently of the sequence of kernels $\left\{K_{j}\right\}_{j}$ given, we always have

$$
L(\log L)^{m} \log \log \log L \subset \mathcal{L}_{m},
$$

with inclusion continuous in the norm.

As before, the case $m=0$ leads to a better result.

THEOREM 16. For any sequence of kernels $\left\{K_{j}\right\}_{j}$ given, we always have

$$
L^{1}=\mathcal{L}_{0}, \quad\|\cdot\|_{L^{1}} \sim\|\cdot\|_{\mathcal{L}_{0}} .
$$

These results are simple reflections of the approximating result in Section 2 (Lemma 5). It seems plausible that $\mathcal{L}_{m}=L(\log L)^{m}$, but this is far from being established, even if we replace in the definition of $\mathcal{L}$ the $L^{1}$-norm in (21) by the weaker "norm" of $L^{1, \infty}$ (Theorem 14 is still true with this definition of $\mathcal{L}$, of course).

Let us point out that once we have fixed the class $B$, the process of defining $\mathcal{L}$ cannot be iterated (so that we would obtain an increasing sequence of 
"better" spaces). In fact, it is very easy to see that $[\mathcal{L}]=\mathcal{L}$. The remarkable observation made by Antonov is that for the a.e. convergence of Fourier series it is still possible to obtain a non-trivial self-improvement result like the one described (replacing the $L^{1}$-norm in $(21)$ by the $L^{\infty}$-norm in this case) and which corresponds to the case $m=1$ in Theorem 15 .

Starting with inequality (20) for a certain space $Q$, Arias de Reina [2] has constructed another space, $Q A$, so that $S^{*}$ (Carleson's maximal operator) maps $Q A$ boundedly into $L^{1, \infty}$. It is easily seen, by that construction, that $Q A \subset[Q]_{\infty}$, where $[Q]_{\infty}$ is the corresponding space defined by replacing the $L^{1}$-norm in (21) with the $L^{\infty}$-norm. It turns out that $Q$ happens to be equal to the space $B_{1}^{*}$ defined in [11], so that $Q A \subset\left[B_{1}^{*}\right]=\mathcal{L}_{1}$. The proof of Theorem 14 shows that the boundedness of $S^{*}$ still holds on the larger space $\left[B_{1}^{*}\right]_{1, \infty}$ (the one defined by replacing the $L^{1}$-norm in (21) with the $L^{1, \infty}$-norm), although it might just happen that the three spaces, $Q A, \mathcal{L}_{1}$ and $\left[B_{1}^{*}\right]_{1, \infty}$, all coincide. It would be very interesting to study the functional-analytic properties of the spaces [.] to see how far one can go in this direction. In the case of the partial sums of Fourier series, this amounts to studying the approximating properties of the Dirichlet kernels. We plan to study some of the questions raised here and give the details of the statements presented in this section in a forthcoming paper.

\section{References}

[1] N. Yu. Antonov, Convergence of Fourier series, East J. Approx. 2 (1996), 187-196.

[2] J. Arias de Reina, Pointwise convergence of Fourier series, J. London Math. Soc. (2) 65 (2002), 139-153.

[3] C. Bennett and R. Sharpley, Interpolation of Operators, Pure Appl. Math. 129, Academic Press, 1988.

[4] L. Carleson, On convergence and growth of partial sums of Fourier series, Acta Math. 116 (1966), 135-157.

[5] M. J. Carro and J. Martín, Endpoint estimates for restricted inequalities, preprint.

[6] M. de Guzmán, Differentiation of Integrals in $\mathbb{R}^{n}$, Lecture Notes in Math. 481, Springer, 1975.

[7] R. Hunt, On the convergence of Fourier series, in: Orthogonal Expansions and their Continuous Analogues (Edwardsville, IL, 1967), Southern Illinois Univ. Press, 1968, 235-255.

[8] P. Sjölin, An inequality of Paley and convergence a.e. of Walsh-Fourier series, Ark. Mat. 7 (1968), 551-570.

[9] - Convergence almost everywhere of certain singular integrals and multiple Fourier series, ibid. 9 (1971), 65-90.

[10] F. Soria, Note on differentiation of integrals and the halo conjecture, Studia Math. 81 (1985), 29-36.

[11] -, On an extrapolation theorem of Carleson-Sjölin with applications to a.e. convergence of Fourier series, ibid. 94 (1989), 235-244. 
[12] E. Stein and G. Weiss, Fourier Analysis on Euclidean Spaces, Princeton Univ. Press, 1971.

[13] A. Zygmund, Trigonometric Series, Cambridge Univ. Press, 1959.

Department of Mathematics

Royal Institute of Technology

S-100 44 Stockholm, Sweden

E-mail: pers@math.kth.se
Department of Mathematics, C-XV

Universidad Autónoma de Madrid

E-28049 Madrid, Spain

E-mail: fernando.soria@uam.es

Received December 16, 2002 